\title{
Methods of estimation of mitral valve regurgitation for the cardiac
} surgeon

\author{
Efstratios E Apostolakis and Nikolaos G Baikoussis*
}

\author{
Address: Cardio-Thoracic Surgery Department, School of Medicine, University Hospital of Patras, Patras, Greece \\ Email: Efstratios E Apostolakis - stratisapostolakis@yahoo.gr; Nikolaos G Baikoussis* - ngbaik@yahoo.com \\ * Corresponding author
}

Published: 15 July 2009

Received: I May 2009

Journal of Cardiothoracic Surgery 2009, 4:34 doi:10.1 186/1749-8090-4-34

Accepted: 15 July 2009

This article is available from: http://www.cardiothoracicsurgery.org/content/4/I/34

(C) 2009 Apostolakis and Baikoussis; licensee BioMed Central Ltd.

This is an Open Access article distributed under the terms of the Creative Commons Attribution License (http://creativecommons.org/licenses/by/2.0), which permits unrestricted use, distribution, and reproduction in any medium, provided the original work is properly cited.

\begin{abstract}
Mitral valve regurgitation is a relatively common and important heart valve lesion in clinical practice and adequate assessment is fundamental to decision on management, repair or replacement. Disease localised to the posterior mitral valve leaflet or focal involvement of the anterior mitral valve leaflet is most amenable to mitral valve repair, whereas patients with extensive involvement of the anterior leaflet or incomplete closure of the valve are more suitable for valve replacement. Echocardiography is the recognized investigation of choice for heart valve disease evaluation and assessment. However, the technique is depended on operator experience and on patient's hemodynamic profile, and may not always give optimal diagnostic views of mitral valve dysfunction. Cardiac catheterization is related to common complications of an interventional procedure and needs a hemodynamic laboratory. Cardiac magnetic resonance (MRI) seems to be a useful tool which gives details about mitral valve anatomy, precise point of valve damage, as well as the quantity of regurgitation. Finally, despite of its higher cost, cardiac MRI using cine images with optimized spatial and temporal resolution can also resolve mitral valve leaflet structural motion, and can reliably estimate the grade of regurgitation.
\end{abstract}

\section{Introduction}

The classical indications for surgical intervention of patients with mitral regurgitation are based either on the symptoms, or on the function of left ventricle and the estimated degree of regurgitation in the non-symptomatic patients [1]. According to the 2007 guidelines of American Heart Association [2] mitral valve (MV) surgery is recommended: 1. for symptomatic patients with acute severe mitral regurgitation (MR). 2. MV surgery is beneficial for patients with chronic severe MR and NYHA functional class II, III, or IV symptoms in the absence of severe left ventricle (LV) dysfunction (severe LV dysfunction is defined as ejection fraction less than 0.30) and/or endsystolic dimension greater than $55 \mathrm{~mm}$. 3. MV surgery is beneficial for asymptomatic patients with chronic severe MR and mild to moderate LV dysfunction, ejection fraction 0.30 to 0.60 , and/or end-systolic dimension greater than or equal to $40 \mathrm{~mm}$. 4 . MV repair is recommended over MV replacement in the majority of patients with severe chronic MR who require surgery, and patients should be referred to surgical centers experienced in MV repair [2]. In the cases of ischemic mitral regurgitation, the decision to operate the mitral valve in combination with bypass grafting is more difficult, and should generally be made preoperatively. According to the ACC/AHA guidelines, it is indicated if the severity of regurgitation is characterized "severe", namely $3+$ or $4+$, and also a significant left ventricular dysfunction is evident [2]. Bolling S. 
reported in his article that a vicious cycle of continuing volume overload, ventricular dilation, progression of annular dilation, increased $\mathrm{LV}$ wall tension and worsening MR and heart failure occur [3]. In other words, in every case of mitral regurgitation, the indication for surgical intervention is based on a reliable quantification of several paraclinical methods. How reliable are these methods? Three are the methods of preoperative estimation of mitral regurgitation: cardiac catheterization, Doppler echocardiography and magnetic resonance imaging (MRI). We would like to compare these diagnostic methods and the information which provide each of them to cardiac surgeon.

\section{A) Cardiac catheterization}

Valvular regurgitation can be evaluated by angiography. Angiographic evaluation of regurgitant severity is based on ejection of contrast media into the left atrium, through the affected mitral valve, or into the left ventricle through the insufficient aortic valve [1]. The severity of regurgitation is graded on a semi quantitative scale of $0+$ to $4+$ (see table 1).

\section{Severity of mitral valve regurgitation}

It is evident from table 1 , that the distinction between the 4 several grades of regurgitation are difficult and in most cases inaccurate. This mode of estimation of degree of regurgitation has some important limitations, which confutes its usefulness: a) the quantity of contrast material (volume and speed of injection) is proportional of density and if this is small may downregulate the grade of regurgitation $[4,5], b)$ the arrhythmia (ventricular extra-beats or atrial fibrillation, or even that produced by the catheter itself) significantly affects the ventricular filling and subsequently the indicated grade of regurgitation[5], c) although mild regurgitation is clearly distinct from severe regurgitation, intermediates grades may not be reliable estimated [1], d) the position of catheter in the ventricle (for mitral valve) or in the aorta (for aortic valve), in relation to the site of valve[5], e) the recorded plane of ventricle and/or atrium, to avoid overlapping. The "ideal" plane for estimation of aortic regurgitation is that of $45^{\circ}$ in left anterior oblique view with $10-15 \%$ of cranial angulation, while that for mitral regurgitation is a $30^{\circ}$ in right anterior oblique view [1]. f) avoid the overlapping of descending thoracic aorta and left atrium which may overestimate the mitral regurgitation $[1], g$ ) avoid derangements of preload and afterload (systemic and pulmonary vascular resistance for aortic and mitral valve, respectively) which significantly affects the grade of regurgitation, h) the coexistence of mitral and aortic regurgitation can change the regurgitant contrast volume through the mitral valve and therefore overestimates the grade of its regurgitation [6]. According to Otto C [6], angiography offers evaluation of grade of regurgitation only "in selected cases", and especially when the non-invasive evaluation is inconsonant to the clinical findings. The advantages and disadvantages of angiography, Doppler and MRI are presented in table 2.

Advantages and disadvantages of angiography, Doppler and MRI Another significant component of estimation of valve regurgitation is the calculation of regurgitant volume and fraction. Regurgitant volume can be calculated by the formula: regurgitant SV = total SV-forward SV, where total SV is the total amount of ejected blood by the LV, measured from left ventricular angiogram, and forward SV the amount of blood ejected through the aortic valve, and measured by Fick's thermodilution technique [6]. According to this equation, may be measured the regurgitation fraction which characterizes the severity of valve regurgitation: for $<20 \%$ mild, $20-40 \%$ moderate, $40-60 \%$ moderately severe, and $>60 \%$ severe regurgitation [5]. Unfortunately, this method also has its limitations. First,

Table I: Angiographic grading of regurgitant severity of aortic and mitral valve [I].

\begin{tabular}{lll}
\hline Grade & Aortic regurgitation & Mitral regurgitation \\
\hline $1+\quad \begin{array}{l}\text { Contrast refluxes from the aortic root into the left ventricle but } \\
\text { clears on each beat }\end{array}$ & Contrast refluxes into the left atrium but clears on each beat \\
\hline $2+\quad$ & $\begin{array}{l}\text { Contrast refluxes into the left ventricle with a gradually } \\
\text { increasing density of contrast in the left ventricle that never equals } \\
\text { contrast intensity in the aortic root }\end{array}$ & $\begin{array}{l}\text { Left atrial contrast density gradually increases but never equals } \\
\text { left vente density }\end{array}$ \\
\hline $3+\quad \begin{array}{l}\text { Contrast refluxes into the left ventricle with a gradually } \\
\text { increasing density such that left ventricle and aortic root density } \\
\text { are equal after several beats }\end{array}$ & $\begin{array}{l}\text { The density of contrast in the atrium and ventricle equalize after } \\
\text { several beats }\end{array}$ \\
\hline $4+\quad \begin{array}{l}\text { Contrast fills the left ventricle resulting in an equivalent } \\
\text { radiographic density in the left ventricle and aortic root on the } \\
\text { first beat }\end{array}$ & $\begin{array}{l}\text { The left atrium becomes as dense as the left ventricle on the first } \\
\text { beat and contrast is seen refluxing into the pulmonary veins }\end{array}$ \\
\hline
\end{tabular}

The points of obscureness are in bold or with questionmarks. 
Table 2: Advantages and disadvantages of three methods of estimation of left-sided valve's regurgitation.

\begin{tabular}{|c|c|c|}
\hline Mode of evaluation & Advantages & Disadvantages \\
\hline Angiography & $\begin{array}{l}\text {-simultaneous calculation of SVR, PVR, LVEDP, PCWP, } \\
\text { EF, etc }(1,6) \\
\text {-easy interpretation by cardiologists and cardiac } \\
\text { surgeons }(6)\end{array}$ & $\begin{array}{l}\text {-invasive method, risk of complications }{ }^{(5)} \\
\text {-misinterpretation in double valve disease }(1,6) \\
\text {-higher cost }(5,6) \\
\text {-temporarily affects hemodynamic of patient (SVR, PVR) and } \\
\text { obscure the results (5) } \\
\text {-time-consuming }\end{array}$ \\
\hline Doppler & $\begin{array}{l}\text { - non-invasive method } \\
\text { - no risks } \\
\text { - low-cost } \\
\text { - time-consuming } \\
\text { - does not affect hemodynamically the patient } \\
\text { - semi-quantitative }\end{array}$ & $\begin{array}{l}\text { - overlapping structures } \\
\text { - "bad" window } \\
\text { - operator depending } \\
\text { discomfort+hemodynamic interaction of TEE - limitations } \\
\text { (see text) } \\
\text { - influence of site of Jet }\end{array}$ \\
\hline MRI & $\begin{array}{l}\text { - measurement of LVEDV, LVESV, LV mass } \\
\text { - no risk } \\
\text { - non invasive tool } \\
\text { - precise and valid estimation }(23,24) \\
\text { - does not need suppression or anaesthesia } \\
\text { (such as TEE) } \\
\text { - estimation of myocardial function and viability }(9,29)\end{array}$ & $\begin{array}{l}\text { - respiratory interference } \\
\text {-not-hemodynamic measurement } \\
\text { - not-anatomic information } \\
\text { - discomfort for the patient } \\
\text { - artefacts in the case of metallic materials }(40,41)\end{array}$ \\
\hline
\end{tabular}

(SVR: systemic venous resistance, PVR: pulmonary venous resistance, PCWP: pulmonary capillary wedge pressure, EF: enjection fraction, LVEDV: left ventricle end diastolic volume), LVESV: left ventricle end systolic volume, LV mass: left ventricle mass, TEE: Transesophageal echocardiography).

its accuracy is depended on the accuracy of measurements of cardiac output [6], and second, in the case of coexistence aortic and mitral regurgitation, only a rough estimation of the portion of regurgitant fraction of each valve can be made [6].

\section{B) Doppler Echocardiography}

Mitral regurgitation is a relatively common and important heart valve lesion in clinical practice and adequate assessment is fundamental to decisions on management. Echocardiography is the recognized investigation of choice for mitral valve regurgitation $[7,8]$. However, the technique is operator dependent and may not always give reliable diagnostic views for estimation of mitral valve dysfunction. Transesophageal echocardiography, with 3dimentional visualization if available, generally gives a better overall assessment of mitral valve dysfunction and the lesions responsible for it, but is also operator dependent, semi-invasive and usually requires patient's sedation [9]. The latter may affect the quantity of mitral regurgitation especially in the cases of ischemic origin. According to Aklog L [10], 90\% of their patients with moderate $(3+/$ $4+)$ mitral regurgitation who underwent intraoperative TEE had their MR downgraded to mild or less $(1+-2+/ 4+)$, and in $30 \%$ of their patients, there was no detectable MR on intraoperative TEE. Similar results reported and other studies concerning the influence of anaesthesia and sedation on downgrading of real mitral regurgiration $[11,12]$. It is recognised that TEE assesses the mechanisms of valve dysfunction well (leaflet prolapse/restriction) and is perhaps the technique best able to determine the structural lesion responsible for the incompetence (chordal/papillary muscle rupture/elongation, leaflet perforation, etc). Although TTE images using harmonic imaging can usually identify leaflet abnormalities in mitral valve prolapse, many patients will have poor image quality due to, reduced ultrasound penetration through scar tissue, air filled lung or excess adipose tissue [13]. Because of variation in image quality and imaging widows systematic segmental mapping of the mitral valve leaflets is often not attempted using 2-dimensional TTE in clinical practice. The standard echocardiographic examination generally in a valvular disease is based on Doppler colour flow imaging, on pulsed Doppler transvalvular velocities, and on continuous wave Doppler measures of regurgitant severity [14]. Doppler colour flow imaging is used to estimate the severity of aortic or mitral valve regurgitation. The amount of regurgitant jet within the antecedent chamber (namely $\mathrm{LV}$ for the aortic regurgitation and left atrium for the mitral regurgitation), is directly proportional to the severity of valve regurgitation [15]. However, there are also included some important limitations in this method: a) a small degree of mitral and aortic valve regurgitation is seen in $70-80 \%$, and in $5-10 \%$ respectively, of normal individuals [14], b) typically the size of the jet is indexed to the size of the left atrium, and it is a drawback of this method for precise estimation of mitral regurgitation [15]. C) The site of jet affects the measured grade of regurgitation. Jets that are peripheral or impinging on a wall, rather than centrally, cause underestimation of severity of regurgitation (of regurgitant volume) up to $40 \%[16,17]$. Quantification of mitral regurgitation is also heavily 
dependent on the colour Doppler flow area, and pertains to the holosystolic regurgitation [15]. The criteria of characterization of severity of mitral regurgitation are included in the table 3 .

\section{Criteria of characterization of severity of mitral regurgitation} It is obvious from table 3: a) that the distinction between mild and severe grade of regurgitation by using the method is easy, but not for the intermediate (II and III) grades, and b) the measurement all of above mentioned parameters of Doppler ECHO is dependent on many others hemodynamic paramenters such as the preload, afterload, and rhythm [14], anatomic parameters such as the dimensions of left atrium [15], or on technical parameters such the "window" [14], as well as on other parameters such as operator's experience, ability of device, etc. For these reasons, interpretation of colour flow data is quite variable, with a disagreement of $29 \%$ for aortic and $25 \%$ for mitral regurgitation $[14,18]$.

There are also some others proposed semi-quantitave mitral regurgitation indices, such as a scoring system of severity, with included most of parameters of table 3, and scored each of them between 0 and $3[17,19]$. According to this system, the total score is divided through the number of evaluated parameters, and for index $>2.2$ indicates a severe regurgitation, $>1.7$ a mild, and index from 1.7 to 2.2 indicate an intermediate or better, vague estimation of regurgitation. However, and this method has the same limitations which reported earlier, plus that of values approximation (methodological problems).

According to Loick et al [20], the intraoperative echocardiographic assessment of mitral regurgitation is reliable, simple and relatively unaffected by hemodynamics. It means that in the one side, it may be involved fewer imponderable factors, but on the other side, it may not be acceptable from a surgical point of view, because it may underestimate the grade of regurgitation $[14,20,21]$.

The regurgitant volume is estimated by using the proximal isovelocity surface area (PISA) on colour Doppler imaging $[22,23]$. This measurement has also several important limitations such as the integration of diastolic flow, as well as caveats of used mathematic types [14].

\section{C) Magnetic Resonance Imaging}

Magnetic Resonance Imaging is a third method for estimation (quantification) of mitral valve regurgitation. The assessment is based on estimation of regurgitant volume by determining the difference between the stroke volumes of ventricles [24]. Stroke volumes are calculated from a stack frame of images as the difference between anddiastolic and end-systolic volumes for each ventricle [24]. In the normal individuals, stroke volume of right ventricle is nearly equivalent to that of left ventricle. Every difference between the two measured stroke volumes indicates the amount of blood which comes back through the insufficient valve during diastole. The estimation is precise, but the limitations of method are the following: a) the measurement is reliable only for the case of single regurgitant valve; in the cases of combined aortic and mitral regurgitation, the difference represents the sum of regurgitant volume [23]. b) The estimation is valid, only if the tricuspid valve is sufficient $[23,24]$. Another method for quantification of valve regurgitation is the cine MRI $[24,25]$. Especially for the aortic valve, this method can discriminate between antegrade and retrograde flow during the cardiac cycle by analysis of bright or dark voxels in the ascending aorta, enabling retrograde flow to be directly measured $[24,26]$. Diastolic retrograde aortic flow equals aortic regurgitant volume, and correlates closely with volumetric cine MRI [23,24].

Table 3: The severity of mitral regurgitation according to the Doppler echocardiography ([15]

\begin{tabular}{|c|c|c|c|c|}
\hline Severity Of Mitral Valve Regurgitation & I (mild) & II & III & IV (severe) \\
\hline Jet (\% Left atrium) & $<15 \%$ & $15-30 \%$ & $35-50 \%$ & $>50 \%$ \\
\hline Spectral Doppler & Faint & - & - & Dense \\
\hline Vena contracta & $<3 \mathrm{~mm}$ & - & - & $>6 \mathrm{~mm}$ \\
\hline Pulmonary vein flow & $S>D$ & - & - & Systolic reversed \\
\hline Right ventricle (ml) & $<30$ & $30-45$ & $45-59$ & $>60$ \\
\hline Effective regurgitant orifice (ERO in $\mathrm{cm} 2$ ) & $<0.20$ & $0.20-0.29$ & $0.30-0.39$ & $>40$ \\
\hline Proximal Isovelosity Surface Area (PISA) & small & - & - & large \\
\hline
\end{tabular}

The points of inaccuracy are depicted in bold or with question marks. 
According to Kizilbash et al [27], the MRI provides accurate measurements of regurgitant flow that correlate well with quantitative Doppler imaging, and in addition, it is the most accurate non-invasive technique for measuring ventricular end-diastolic volume, end-systolic volume and left ventricular mass. Concerning the estimation of mitral regurgitation MRI in the last years is considered more reliable in comparison to these of echocardiography. In fact, there are many studies comparing the two methods $[7,8]$. Cardiovascular MRI has many advantages like accurate determination of left as well as right ventricular volumes and function $[28,29]$, measurements of aortic flow volume, and in ischemic mitral regurgitation, comprehensive assessment of regional myocardial function and viability $[28,30]$. When used optimally, MRI can complement echocardiography in the assessment of mitral regurgitation, especially in patients in whom transthoracic echocardiography has not provided adequate information [9]. Finally, MRI has been proved that overtake the limitations of ecocardiography (overlapping structures, "bad window", artefacts, or contraindications of TEE. On the other hand, in its limitations are included the respiration, and the higher cost [7].

The mitral regurgitant volume (MRV) measured by MRI is the difference between the LV stroke volume (LVSV) and the aortic forward stroke volume (AoSV) i.e. MRV (mls/ beat $)=$ LVSV - AoSV. The regurgitant fraction (RF) is the ratio of the MRV divided by the LVSV i.e. $\mathrm{RF}(\%)=(\mathrm{MRV}$ $\times$ LVSV) $\times 100[9]$.

It may also be possible to directly measure mitral inflow volume by phase-contrast velocity flow mapping at the tips of the mitral valve leaflets but this requires a specialised imaging sequence which tracks the motion of the mitral valve annulus during the cardiac cycle [31]. In the absence of other regurgitant lesions, MRV can also be calculated by subtracting the right ventricle stroke volume (RVSV) from the LVSV i.e. MRV = LVSV - RVSV, using established techniques [28]. However, the calculation of RVSV is less reproducible compared to LVSV due to the extensive trabeculation of the right ventricle (RV). Moreover, associated tricuspid regurgitation is reported in up to $50 \%$ of patients with significant mitral regurgitation and this invalidates the use of RVSV to determine MRV [32]. The American College of Cardiology $[2,8]$ has established echocardiographic criteria for grading the severity of mitral regurgitation. In the absence of established criteria for MRI, the findings of this study, derived from LV volume and ascending aortic flow measurements, can be noted: mild $=\mathrm{RF} \leq 15 \%$, moderate $=\mathrm{RF} 16-24 \%$, moderate-severe $=$ RF $25-42 \%$, severe $=R F>42 \%$.

A further feature of severe mitral regurgitation is reversal of flow in the pulmonary veins during LV systole, which may be visible in the 4 chamber and certain mitral stack cines [9].

Evaluation of mitral valve dysfunction from standard, routinely acquired MRI imaging planes alone is rarely adequate. The proposed technique by Kim RJ et al [9], with additional imaging of the mitral valve based on its anatomy, allows more detailed evaluation of its dysfunction.

In degenerative valve disease, MRI allows determination of the leaflet scallop, responsible for the valve dysfunction e.g. P2 or P3 prolapse, and hence helps guide surgical repair. In rheumatic valve disease, ÌRI allows assessment of the severity of valve restriction and hence helps determine the feasibility of valve repair and the need for valve replacement. In functional mitral regurgitation due to ischemic heart disease or cardiomyopathy, it confirms the diagnosis and helps exclude coexisting degenerative valve disease. Comparison of the accuracy and reproducibility of MRI using this technique with echocardiography, especially transesophageal echocardiography, and findings at surgery will need to be done. Two recent studies using similar techniques as described here, but without the additional slices taken at the commissural ends of the mitral valve, have recently been published $[33,34]$. The first study reported a sensitivity and specificity of $89 \%$ and $88 \%$ respectively for detecting flail or prolapsed leaflets compared to findings at surgery in 47 patients. This compared with a sensitivity and specificity of $93 \%$ and $88 \%$ respectively for transesophageal echocardiography [33]. The second study reported agreement between CMR assessment and transthoracic echo determination of prolapsed or flail leaflets in $92 \%$ of 27 patients [33]. According to the lattest study, there was an excellent concordance between MRI and transthoracic echocardiography in the identification of jet direction and leaflet abnormality. MRI mapping of the mitral valve using a simple protocol can reliably acquire long axis images through the valve, facilitating localisation of leaflet abnormalities and regurgitant jet direction. When compared to modern TTE, the MRI mapping protocol accurately identified the abnormal leaflet in $98 \%$ of cases [34]. The difference between the 2 techniques was differentiating leaflet flail from prolapse in 3 patients and MRI failing to detect a borderline prolapse (2 $\mathrm{mm}$ ) involving an anterior mitral valve leaflet. Using either technique, variation in defining the border between adjacent leaflet segments (e.g. A1 from A2) can lead to minor differences in classification but is less likely to effect the decision for valve repair versus replacement. The presence of a flail mitral valve leaflet identifies patients who are at a higher risk of sudden cardiac death and may warrant early surgery if the valve is repairable [35]. The discrepancies in classification of prolapse and flail segments may also in part be due to superior spatial resolution of echocardiographic over MRI when there are 
adequate echocardiographic windows. MRI spatial resolution is dependent on the voxel size and the slice thickness of the planes used. Hence, visualising the direction of the mitral valve leaflet tip ( $1-5 \mathrm{~mm}$ thickness dependent on the degree of mitral leaflet thickening) to define segment prolapse versus flail may be difficult. In addition, insufficient contrast between the signal loss defining the origin of the regurgitant jet and the distal mitral leaflet tip may contribute to the minor differences seen. An advantage of MRI compared to TTE is that because there is no limitation of imaging windows the MRI mapping protocol enabled a complete and systematic assessment of the mitral valve in every patient. Acquisition of the mapping images is usually efficient, requiring on average 7 cine images, and between 5 to 10 minutes per patient. MRI is an accurate, reproducible, and non-invasive manner, potentially enabling better estimation of the timing and type of surgical intervention. Cine MRI is a highly sensitive diagnostic tool to assess changes in LV mass and volume [36-39]. This is supported by Bottini et al. They showed that MRI is the most precise method for measuring LV mass when compared to Transthoracic echocardiography in hypertensive patients [40], suggesting that for more specific questions MRI may be the more reliable imaging tool. Francois et al. have shown that MRI assessment of LV mass correlated well with true LV mass measurements during autopsy [41]. However, MRI is expensive, time-consuming, and only available in specialized centers and therefore no alternative for routine patient follow-up in smaller hospitals and private practices.

\section{Conclusion}

In patients with mitral valve regurgitation MRI has an established role in the assessment of LV size and function and mitral regurgitation severity. With the addition of mitral valve mapping, MRI can potentially provide a comprehensive assessment of mitral regurgitation. Comprehensive assessment of mitral regurgitation requires assessment of: (a) its severity to determine the need for surgical intervention, (b) the mechanism of the dysfunction to determine the type of surgical intervention required (leaflet prolapse/restriction, including the leaflet scallops involved: A1-P1, A2-P2, A3-P3); (c) LV volumes and function to determine the timing and risks of surgery; and, in ischemic mitral regurgitation, (d) LV viability. Such comprehensive assessment is feasible in a single MRI examination but needs a defined protocol, as described in this paper. When used optimally, MRI can complement existing imaging modalities such as echocardiography in the assessment of patients with mitral regurgitation. The fixed imaging planes of MRI and its suboptimal throughplane resolution rarely permit adequate visualisation of the chordal structures to identify rupture or elongation accurately. MRI is also not suited for visualisation of leaflet and annular calcification which are important factors influencing the probability of successful valve repair. MRI, when used optimally, may therefore play a useful role in assessing the mitral valve in patients in whom transthoracic echocardiography has not provided adequate imaging and in whom transesophageal echocardiography is considered too invasive.

\section{Competing interests}

The authors declare that they have no competing interests.

\section{Authors' contributions}

All authors: 1. have made substantial contributions to conception and design, or acquisition of data, or analysis and interpretation of data; 2 . have been involved in drafting the manuscript or revisiting it critically for important intellectual content; 3 . have given final approval of the version to be published.

\section{References}

I. Otto C: Valvular Heart Disease. 2nd edition. Saunders an Imprint of Elsevier; 2004:404-5.

2. Endorsed by the Society for Cardiovascular Angiography and Interventions Developed in Collaboration With the Society of Cardiovascular Anaesthesiologists 1998 Guidelines for the Management of Patients With Valvular Heart Disease): Association Task Force on Practice Guidelines (Writing Committee to Revise the Disease: A Report of the American College of Cardiology/American Heart ACC/ AHA 2006: Guidelines for the Management of Patients With Valvular Heart. Circulation 2007, I I 5(I 5):e409.

3. Bolling $\mathrm{S}$ : Mitral valve reconstruction in the patients with heart failure. Heart Failure reviews 2001:177-185.

4. Gorman R, Gorman J, Edmunds H: Ischemic mitral regurgitation. In Cardiac Surgery in the Adult 2nd edition. Edited by: Cohn L, Edmunds H. MacGraw Hill; 2003:762.

5. Grossman W: Profiles in valvular heart disease. In Grossman's Cardiac Catheterization, Angiography and Intervention 6th edition. Edited by: Baim D, Grossman W. Williams and Wilkins; 2000:759-84.

6. Otto C: Valvular Heart Disease. 2nd edition. Saunders An Imprint of Elsevier; 2004: I I I-I I2.

7. Fogel M: Cardiac Magnetic Ressonance Imaging. In Mastery in Cardiothoracic Surgery 2nd edition. Edited by: Kaiser L, Kron I, Spray T. Lippincot Williams and Wilkins; 2007:66I. and 675

8. American College of Cardiology/American Heart Association TaskForce on Practice Guidelines; Society of Cardiovascular Anesthesiologists; Society for Cardiovascular Angiography and Interventions; Society of Thoracic Surgeons, Bonow RO, Carabello BA, Kanu C, de Leon AC Jr, Faxon DP, Freed MD, Gaasch WH, Lytle BW, Nishimura RA, O'Gara PT, O'Rourke RA, Otto CM, Shah PM, Shanewise JS, Smith SC Jr, Jacobs AK, Adams CD, Anderson JL, Antman EM, Faxon DP, Fuster V, Halperin JL, Hiratzka LF, Hunt SA, Lytle BW, Nishimura $R$, Page RL, Riegel B: ACCIAHA 2006: guidelines for the management of patients with valvular heart disease: a report of the American College of Cardiology/American Heart Association Task Force on Practice Guidelines (writing committee to revise the 1998 Guidelines for the Management of Patients With Valvular Heart Disease): developed in collaboration with the Society of Cardiovascular Anesthesiologists: endorsed by the Society for Cardiovascular Angiography and Interventions and the Society of Thoracic Surgeons. Circulation 2006, I I 4:e84-23I.

9. Chan J, Wage R, Symmonds K, Rahman-Haley S, Mohiaddin R, Firmin $D$, Pepper J, Pennel D, Kilner P: Towards comprehensive assessment of mitral regurgitation using cardiovascular magnetic resonance. J Cardiovasc Magn Reson 2008, I O(I):6I.

10. Aklog L, Filsoufi F, Flores K, Chen RH, Cohn LH, Nathan NS, Byrne JG, Adams DH: Does coronary artery bypass grafting alone correct moderate ischemic mitral regurgitation? Circulation 200I, 1 04: 168-175. 
II. Grewal K, Malkowski M, Piracha A, Astbury JC, Kramer CM, Dianzumba S, Reichek N: Effect general anesthesia on the severity of mitral regurgitation by transesophageal echocardiography. Am J Cardiol 2000, 85: 199-203.

12. Bach D, Deeb G, Bolling S: Accuracy of intraoperative transesophageal echocardiography for estimating the severity of functional mitral regurgitation. $A m$ J Cardiol 1995, 76(7):508-5I2.

13. Monin JL, Dehant P, Roiron C, Monchi M, Tabet JY, Clerc P, Fernandez G, Houel R, Garot J, Chauvel C, Gueret P: Functional assessment of mitral regurgitation by transthoracic echocardiography using standardized imaging planes diagnostic accuracy and outcome implications. J Am Coll Cardiol 2005, 46:302-309.

14. Gillinov M, Cohn L, Edmunds H, Eds: Cardiac Surgery in Adults. 2nd edition. 2003:79l.

15. Armstrong W: Echocardiography. In Braunwald's Heart Disease 7th edition. Edited by: Zipes D, Libby P, Bonow R, Braunwald E. Elsevier Saunders; 2005:213-14.

16. Chao K, Moises V, Shandas R, Elkadi T, Sahn DJ, Weintraub R: Influence of the Coand effect on color Doppler jet area and color encoding: In vitro studies using color Doppler flow mapping. Circulation 1992, 85:333-4I.

17. Sugeng L, Spencer K, Mor-Avi V, DeCara JM, Bednarz JE, Weinert L, Korcarz CE, Lammertin G, Balasia B, Jayakar D, Jeevanandam V, Lang RM: Dynamic three-dimentional color flow Doppler: An improved technique for the assessment of mitral regurgitation. Echocardiography 2003, 20:265.

18. Krebill K, Sung H, Tamura T, Chung KJ, Yoganathan AP, Sahn DJ: factors influencing the structure and shape of stenotic and regurgitant jets: an in vitro investigation using Doppler color flow mapping and optical flow visualization. JACC 1989, 13:1672-81.

19. Gottdiener J, Panza J, St John S, Bannon P, Kushner H, Weissman NJ: Testing the test: the reliability of echocardiography in the sequential assessment of mitral regurgitation. Am Heart $J$ 2002, 144:||15-2|.

20. Loick H, Wichter T, Schmidt C: Mitral Valve Disease. In Transesophageal Echocardiography in Anesthesia and Intensive Care Medicine 2nd edition. Edited by: Poelaert J, Skarvank A. BMJ Books; 2004: I I0.

21. Adams D, Filsoufi F, Aklog L, Farivar RS, Byrne JG, Adams DH: Mitral Valve Repair: Ischemic. In Mastery in Cardiothoracic Surgery 2nd edition. Edited by: Kaiser L, Kron I, Spray T. Lippincot Williams and Wilkins; 2007:37I.

22. Chen C, Koschyk D, Brockhoff C, Heik S, Hamm C, Bleifeld W, Kupper W: Noninvasive estimation of regurgitant flow rate and volume in patients with mitral regurgitation by Doppler color mapping of accelerating fllow field. JACC 1993, 21:374-83.

23. Heinle S: Quantitation of valvular regurgitation. In The Practice of Clinical Echocardiography 2nd edition. Edited by: Otto C. Philadelphia, W.B. Saunders; 2002:367-88.

24. Higgins C: Valvular Heart Disease. In Thoracic Imging-Pulmonary and Cardiovascular Radiology Edited by: Webb R, Higgins C. Lippincott Williams and Wilkins; 2005:707-19.

25. Bonow R, Braunwald E: Valvular Heart Disease. In Braunwald's Heart Disease 7th edition. Edited by: Zipes D, Libby P, Bonow R, Braunwald E. Elsevier Saunders; 2005: 1572.

26. Bonow $R$, Carabello $B$, de Leon $A$ : ACC/AHA guidelines for the management of patients with valvular heart disease: $A$ report of the American College of Cardiology/American Heart Association. Task Force on Practice Guidelines (Committee on Management of patients with valvular heart disease). J Heart Valve Dis 1998, 7(6):672-707.

27. Kizilbash A, Handley W, Willett D, Franco F, Peshock RM, Grayburn PA: Comparison of quantitative Doppler with magnetic resonance imaging for assessment of the severity of mitral regurgitation. Am J Cardiol 1998, 81:792-795.

28. Kim RJ, Wu E, Rafael A, Parker MA, Simonetti O, Klocke FJ, Bonow RO, Judd RM: The use of contrast enhanced magnetic resonance imaging to identify reversible myocardial dysfunction. N Eng J Med 2000, 343: 1445-1453.

29. Grothues F, Smith GC, Moon JC, Bellenger NG, Collins P, Klein HU, Pennell DJ: Comparison of interstudy reproducibility of cardiovascular magnetic resonance with two-dimensional echocardiography in normal subjects and in patients with heart failure or left ventricular hypertrophy. Am J Cardiol 2002, 90:29-34.

30. Kim RJ, Wu E, Rafael A, Chen E-L, Parker MA, Simonetti O, Klocke FJ, Bonow RO, Judd RM: The use of contrast-enhanced magnetic resonance imaging to identify reversible myocardial dysfunction. New England Journal of Medicine 2000, 343: I445-I 453.

31. Kozerke S, Schwitter J, Pedersen EM, Boesiger P: Aortic and mitral regurgitation: quantification using moving slice velocity mapping. J Magn Reson Imaging 200I, I 4: I06-II2.

32. Cohen SR, Sell JE, Mclntosh CL, Clarc CE: Tricuspid regurgitation in patients with acquired, chronic, pure mitral regurgitation. I. Prevalence, diagnosis, and comparison of preoperative clinical and memodynamic features in patients with and without tricuspid regurgitation. J Thorac Cardiovasc Surg 1987, 94:48I-487.

33. Stork A, Franzen $O$, Ruschewski $H$, Ruschewski $H$, Detter $C$, Mullerleile K, Bansmann PM, Adam G, Lund GK: Assessment of functional anatomy of the mitral valve in patients with mitral regurgitation with cine magnetic resonance imaging: comparison with transoesophageal echocardiography and surgical results. Eur Radiol 2007, I7(1 2):3189-3198.

34. Gabriel RS, Kerr AJ, Raffel OC, Stewart RA, Cowan BR, Occleshaw $\mathrm{CJ}$ : Mapping of mitral regurgitant defects by cardiovascular magnetic resonance in moderate or severe mitral regurgitation secondary to mitral valve prolapse. J Cardiovasc Magn Resn 2008, 10:16.

35. Grigioni F, Enriquez-Sarano M, Ling LH, Bailey KR, Seward JB, Tajik A], Frye RL: Sudden death in mitral regurgitation due to flail leaflet. J Am Coll Cardiol 1999, 34:2078-2085.

36. Jenkins C, Bricknell K, Hanekom L, Marwick TH: Reproducibility and accuracy of echocardiographic measurements of left ventricular parameters using real-time three-dimensional echocardiography. J Am Coll Cardiol 2004, 44:878-886.

37. Djavidani B, Schmid FX, Keyser A, Butz B, Seitz J, Luchner A, Debl K, Feuerbach S, Nitz WR: Early regression of left ventricular hypertrophy after aortic valve replacement by the Ross procedure detected by cine MRI. J Cardiovasc Magn Reson 2004, 6:1-8.

38. Bottini PB, Carr AA, Prisant LM, Flickinger FW, Allison JD, Gottdiener JS: Magnetic resonance imaging compared to echocardiography to assess left ventricular mass in the hypertensive patient. Am J Hypertens 1995, 8:221-228.

39. Francois CJ, Fieno DS, Shors SM, Finn JP: Left ventricular mass: Manual and automatic segmentation of true FISP and FLASH cine MR images in dogs and pigs. Radiology 2004, 230:389-395.

40. Condon B, Hadley DM: Potential MR hazard to patients with metallic heart valves: the Lenz effect. Journal of Magnetic Resonance Imaging 2000, I 2: I7I- I 76.

4I. Condon B, Hadley DM: Potential MR hazard to patients with metallic heart valves: the Lenz effect. Journal of Magnetic Resonance Imaging 2000, I 2: I7I-176. Europace (2008) 10, 336-346

Publish with BioMed Central and every scientist can read your work free of charge

"BioMed Central will be the most significant development for disseminating the results of biomedical research in our lifetime. "

Sir Paul Nurse, Cancer Research UK

Your research papers will be:

- available free of charge to the entire biomedical community

- peer reviewed and published immediately upon acceptance

- cited in PubMed and archived on PubMed Central

- yours - you keep the copyright 